\title{
Ascites and Adnexal Mass with Raised CA125: How Arduous can be the Path of Diagnosis
}

\author{
Deepika Pannu, ${ }^{1}$ Sunita Malik, ${ }^{1}$ Saritha Shamsunder, ${ }^{1}$ Amar Bhatnagar ${ }^{1}$ \\ 'Department of Obstetrics and Gynecology, VMMC and Safdarjung Hospital, New Delhi, India.
}

\section{ABSTRACT}

Ascites, adnexal mass and elevated CA125 levels almost always suggest advanced ovarian carcinoma. We present a case of a 37 years old multiparous lady who presented with such a classical picture. Radiological picture was suggestive of ovarian carcinoma with peritoneal metastasis. However ascitic fluid cytology was negative for malignant cells. A differential diagnosis of tubercular mass was made. Ascitic fluid was sent for adenosine deaminase test that was negative. Fine needle aspiration cytology failed to reveal any sufficient sample for evaluation. Open laparotomy and biopsy was done that showed granulomas suggestive of tuberculosis. Category one anti tubercular treatment was started and symptoms resolved within one month.

Keywords: adenosine deaminase; ovarian carcinoma; pelvic tuberculosis.

\section{INTRODUCTION}

Ovarian hyper stimulation, meigs's syndrome (ovarian fibroma), pelvic-peritoneal tuberculosis, epithelial ovarian carcinoma present as adnexal mass with ascites and raised CA 125 levels. ${ }^{1-5}$ Brenner tumors and granulosa cell tumors can also be associated with such a picture (pseudo meig's syndrome). ${ }^{1}$ Very rare causes include ovarian capillary hemangioma, enteroovarian fistula etc. The clinical picture of ascites with adnexal mass is present in $42 \%$ of patients of ovarian malignancy. ${ }^{1}$ Radiological features could be very similar further confusing the diagnosis. We present one such diagnostic enigma.

\section{CASE REPORT}

A 37 year old multiparous woman presented with pain abdomen and distension of two weeks duration. There was no history of urinary and bowel problems, no history suggestive of tuberculosis i.e. cough, hemoptysis, fever, night sweats and weight loss. Her family history and past medical history were unremarkable. Her menstrual cycles were regular. Her general physical examination was unremarkable; there was no anemia, generalized lymphadenopathy and chest examination was normal. Abdominal examination revealed ascites with no organomegaly. Gynecological examination revealed a normal sized uterus and cervix. An ill-defined nodular mass of approximately around five to six $\mathrm{cm}$ was felt in the left fornix with nodularity in pouch of douglas and the right fornix was free. Ultrasound report showed a cyst of $62 \times 52 \times 40 \mathrm{~mm}$ size with well-defined margins in the left ovary with internal septae. Uterus and right adnexal were normal. Investigations revealed Serum CA 125 level to be $1105 \mathrm{IU} / \mathrm{ml}(\mathrm{N}<35 \mathrm{IU} / \mathrm{ml})$. Risk of malignancy index (RMI) score was calculated to be 4420 . Rest of the tumor markers were normal.

Contrast enhanced computed tomography (CECT) of abdomen and pelvis revealed septated ascites with multiple enhancing small nodular and plaque

Correspondence: Dr. Deepika Pannu, Department of Obstetrics and Gynecology, VMMC and Safdarjung Hospital, New Delhi, India. Email: deeasrg@gmail.com, Phone: +91901395680. 
like thickening of peritoneum along with thick and enhancing omental thickening with few fine nodular omental deposits and serosal deposits were seen on the surface of sigmoid colon. Uterus and right ovary was normal. Left ovary showed a cystic lesion with thick enhancing wall measuring $5.2 \times 4.4 \times 4.2 \mathrm{~cm}$ with internal septations. Abutting this lesion another cystic lesion with thick enhancing shaggy wall was seen measuring $1.8 \times 1.2 \mathrm{~cm}$. Mild left pleural effusion was present. Few opacities in apical segment of right upper lobe were present. The findings on imaging were highly suggestive of advanced ovarian carcinoma (Figure 1).

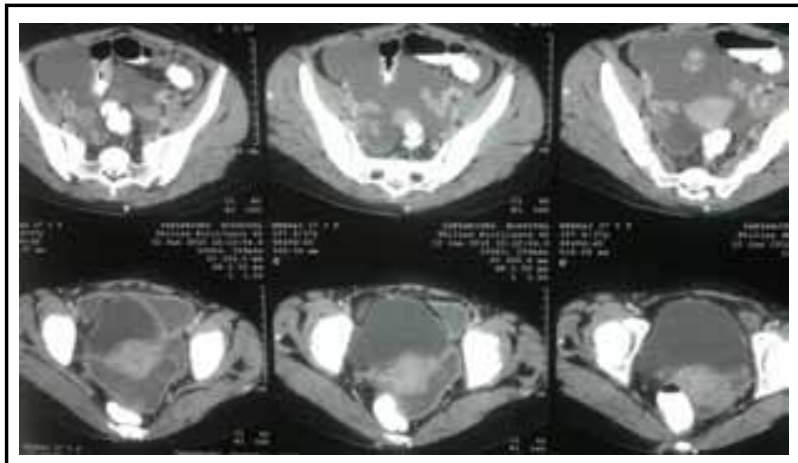

Figure 1. Figure showing septated ascites. Multiple enhancing small nodular thickening of peritoneum seen along with thin plaque like enhancing peritoneal thickening.

We therefore decided to an ascetic tap to confirm the diagnosis in order to start palliative chemotherapy. To our surprise the ascitic fluid analysis revealed a straw coloured fluid which was an exudate on biochemistry. Acid Fast Bacilli were absent and adenosine deaminase (ADA) level from the ascitic fluid was $12 \mathrm{U} / \mathrm{L}$ which was negative. Cytology revealed mononuclear cells only and was negative for malignant cells. FNAC was tried but it failed to produce sufficient sample from omental deposits and peritoneum for evaluation. We were at the cross roads again; we therefore decided for a laparotomy Abdomen was opened with a small infraumblical incision with a possibility of extension if necessary. Straw coloured ascitic fluid of around 2 litres was drained. Left ovary was enlarged and studded with nodules (Figure 2).

Biopsy from multiple sites was taken and abdomen closed. Sections from peritoneal biopsy and omental biopsy showed fibroadipose tissue with numerous epitheloid granulomas and giant cells s/o tuberculosis (Figure 5). Category 1 Anti Tubercular Treatment for severe form of extrapulmonary tuberculosis and the symptoms gradually improved. This patient is well on follow up at 6 months.

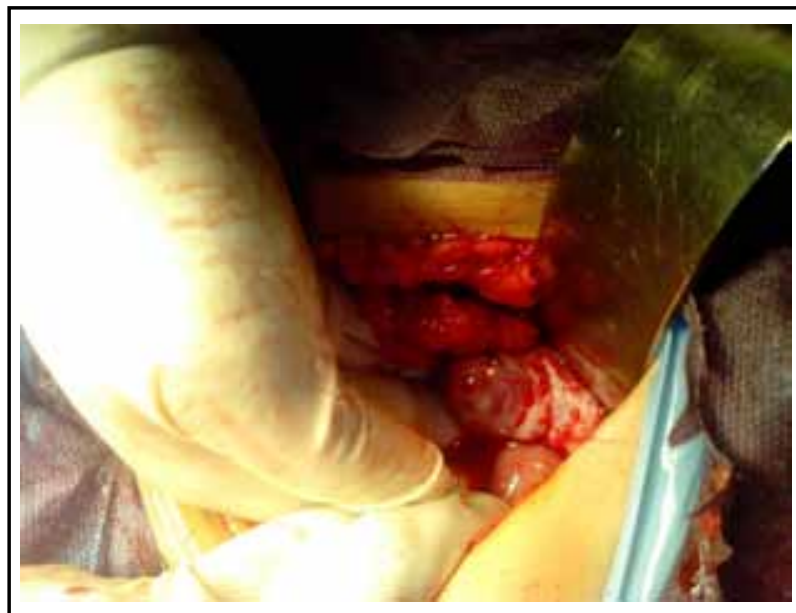

Figure 2. Figure showing peroperative findings showing left ovary.Left ovary was enlarged and right ovary was normal however external surface of both was studded with reddish nodules.

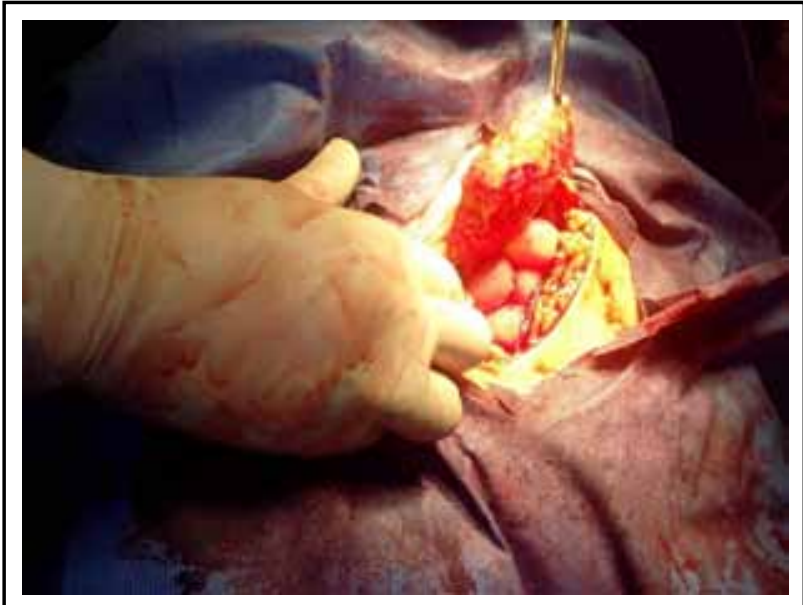

Figure 3. Figure showing nodular omental thickening.

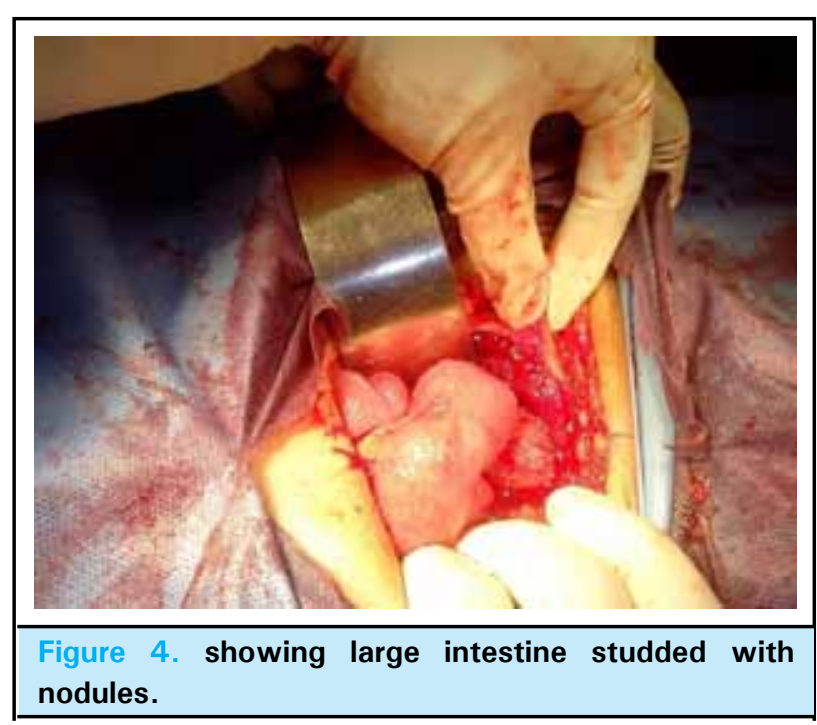




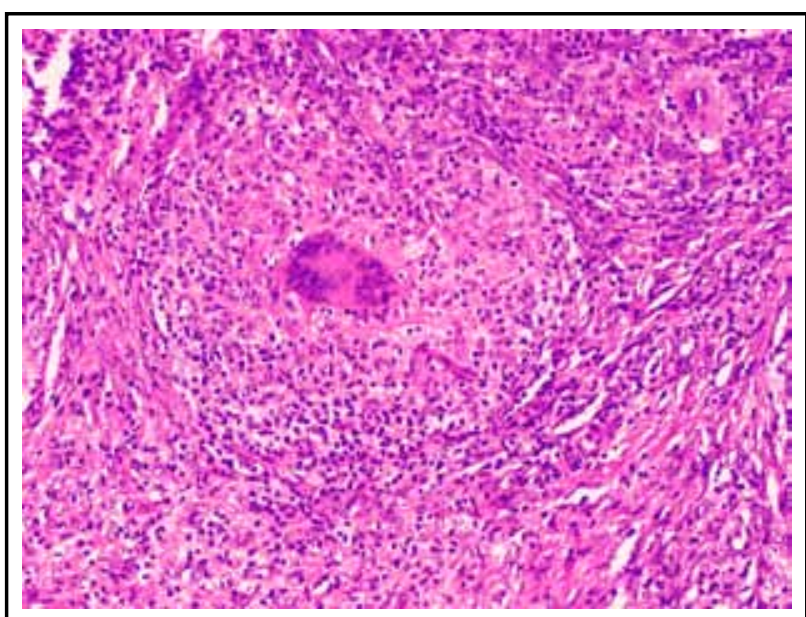

Figure 5. Histopathology slide of peritoneal biopsy revealing the granuloma at the centre.

All abdominal and pelvic organs including peritoneal surfaces were studded with 3-4 $\mathrm{mm}$ nodules (Figure $3,4)$ which were reddish in colour and non caseating.

\section{DISCUSSION}

Our case was a diagnostic dilemma. Very high levels of CA 125 as seen in our case are generally reported in epithelial ovarian carcinoma. It has been stated earlier that levels of CA- $125>65 \mathrm{U} / \mathrm{mL}$ correlate with epithelial ovarian malignancy, this can help distinguish malignant from benign disease with a sensitivity of $75 \%$ to $83 \%$ and specificity of 88 to $92 \% .^{2}$ However case reports of increased CA 125 levels ( $>1000 \mathrm{u} / \mathrm{ml}$ ) are also reported in abdominal tuberculosis. ${ }^{5}$ Further recent reports have shown that serum and ascitic CA-125 levels are elevated in almost all patients with ascites regardless of the underlying cause. ${ }^{6}$ ADA has a role in regulating the level of adenosine and development and maintenance of the immune system. It is involved in proliferation and differentiation of T lymphocytes, malignancies, Collagen vascular diseases and some infections associated with lymphocytosis may increase ADA levels. ${ }^{7}$ It has been shown to have $100 \%$ sensitive and $94.6 \%$ specific for diagnosing tuberculous etiology with positive predictive value of $95.5 \%$ and negative predictive value of $100 \%$ respectively. ${ }^{8}$ Radiological features in tuberculosis and ovarian carcinoma can be very similar. ${ }^{9}$ The nodules of carcinomatosis are generally large, usually more than $3 \mathrm{~mm}$, vascular, and irregular. On the contrary miliary nodules on the peritoneum are each of about 1 to $2 \mathrm{~mm}$ in size, slightly raised and whitish. ${ }^{10}$ Recently Human Epididymis protein 4 (HE4) has emerged out to be a useful marker in differentiating such cases. HE4 is a reliable marker specific to ovaries and epididymis and is not influenced by peritoneal diseases. Normal HE4 in a patient of ascites with adnexal mass strongly suggests peritoneal tuberculosis. ${ }^{11}$

Elevated CA-125 levels in women of reproductive age should prompt consideration of causes other than cancer, especially when serosal fluid is present. Ascitic fluid ADA through said to be the perfect marker for the diagnosis of pelvic tuberculosis, may be false negative. HE4 is emerging tumour marker specific for ovarian cancer. Laparotomy and biopsy may be needed to clinch the diagnosis.

\section{ACKNOWLEDGEIMENTS}

The authors would like to acknowledge the woman for her support and patience and the staff of hospital for their cooperation.

\section{REFERENCES}

1. Shen-Gunther J, Mannel RS. Ascites as a predictor of ovarian malignancy Gynecol Oncol. 2002 Oct;87(1):77-83.

2. Soper JT, Hunter VJ, Daly L. Preoperative serum tumorassociated antigen levels in women with pelvic masses. Obstet Gynecol. 1990;75:249-54.

3. Sugiyama A, Urushihara N, Fukumoto $\mathrm{K}$ et al. Ovarian fibroma with marked ascites and elevated serum levels of CA-125 in a young girl. J Pediatr Surg. 2011 May;46(5):1001-4.

4. Ying Wang, Feng Zhou, Jia-Le Qin et al. Pregnancy luteoma followed with massive ascites and elevated CA125 after ovulation induction therapy: a case report and review of literatures. Int J Clin Exp Med. 2015; 8(1): 1491-1493.
5. D K O'Riordan, A Deery, A Dorman, 0 E Epstein. Increased CA 125 in a patient with tuberculous peritonitis: case report and review of published works. Gut. 1995; 36: 303-305.

6. F. M. Sanai and K. I. Bzeizi, "Systematic review: tuberculous peritonitis-presenting features, diagnostic strategies and treatment," Alimentary Pharmacology and Therapeutics. Vol. 22, no. 8, pp. 685-700, 2005.

7. Yi-Jun Liao, Chun-Ying $\mathrm{Wu}$, Shou-Wu Lee, Chia-Ling Lee, Sheng-Shun Yang, Chi-Sen Chang et al. Adenosine deaminase activity in tuberculous peritonitis among patients with underlying liver cirrhosis. World J Gastroenterol. 2012 Oct $7 ; 18(37)$ : 5260-5265. 
8. Diagnostic value of Adenosine Deaminase (ADA) activity in tubercular serositis. Indian J Tuberc. 2006; 53:92-95.

9. I. Lataifeh , I. Matalka, W. Hayajneh, B. Obeidat, H. Al Zou ' bi \& G. Abdeen. Disseminated peritoneal tuberculosis mimicking advanced ovarian cancer. Journal of Obstetrics and Gynaecology. 2014; Early Online: 1-4.
10. Primary Surgery: Volume One: Non-trauma. The surgery of tuberculosis. Nelson Awori, Anne Bayley 1999 edition.

11. Serum Human Epididymis Protein 4 (HE4) in the Differential Diagnosis of Peritoneal Tuberculosis: A Report of Two Cases. Balkan Med J. 2014 Sep; 31(3): 270-271. 